\title{
Inteligencia fluida y cristalizada en el autismo de alto funcionamiento y el síndrome de fisperger
}

\author{
Fluid and Crystallized Intelligence in High functioning futism and fsperger \\ Syndrome \\ Inteligência fluida e cristalizada no autismo de alto funcionamento e a síndrome \\ de Asperger
}

\author{
Paula Fernanda Pérez Rivero, Lía Margarita Martínez Garrido* \\ Universidad Pontificia Bolivariana, Colombia
}

Doi: dx.doi.org/10.12804/apl33.02.2015.12

\section{Resumen}

La inteligencia en los trastornos del espectro autista, especialmente en el autismo de alto funcionamiento y el síndrome de Asperger, ha sido tema de numerosas investigaciones que pretenden establecer la capacidad intelectual que se observa en estos sujetos. Algunos de los resultados más sobresalientes señalan que la inteligencia fluida es uno de los aspectos de desempeño superior en esta población, en comparación con el desempeño en las pruebas que miden inteligencia cristalizada como la escala Wechsler, razón por la cual los estudios sobre inteligencia deberían utilizar instrumentos que midan el razonamiento abstracto y la capacidad analógica, funciones estrechamente relacionadas con la inteligencia fluida, como lo es el test de matrices progresivas de Raven. En la presente investigación se evaluaron 12 niños con autismo de alto funcionamiento y 6 con síndrome de Asperger, a través de la escala de Wechsler IV y el test de
Raven. Los resultados arrojaron que no existen diferencias significativas entre ambas medidas de inteligencia. No obstante, sí se encontró que existen diferencias en el perfil intelectual de ambos grupos y que el síndrome de Asperger parece tener un nivel intelectual superior, en comparación con el autismo de alto funcionamiento. Palabras clave: autismo; síndrome de Asperger; inteligencia; WISC; Raven.

\section{fbstract}

Intelligence in autistic spectrum disorders, especially high functioning autism and Asperger's syndrome has been the subject of numerous investigations that seek to establish intellectual ability observed in these subjects. Some of the most outstanding results indicate that fluid intelligence is one of the aspects of superior performance in this population compared to tests that measure crystallized intelligence such as the Wechsler

* Paula Fernanda Pérez Rivero, Psicóloga, Especialista en Psicología Clínica, Universidad Pontificia Bolivariana, Colombia. Miembro del Grupo de Neurociencias y Comportamiento UIS-UPB; Lía Margarita Martínez Garrido, Psicóloga, Universidad Pontificia Bolivariana, Colombia. Mestre em Ciências Biológicas y Candidata a Doutora em Ciências Biológicas, Universidade de Brasília, Brasil. Miembro del Grupo de Neurociencias y Comportamiento UIS-UPB.

La correspondencia relacionada con este artículo debe ser enviada a Lía Margarita Martínez Garrido. Correo electrónico: lia.martinez@upb.edu.co

Para citar este artículo: Pérez, P. F. \& Martínez, L. M. (2015). Inteligencia fluida y cristalizada en el autismo de alto funcionamiento y el síndrome de Asperger. Avances en Psicología Latinoamericana, 33(2), 340-359. doi: dx.doi.org/10.12804/ ap133.02.2015.12 
scales, providing a reason for using in intelligence studies instruments that measure abstract reasoning and the analogical capacity, closely related to fluid intelligence function, one of this like the Raven progressive matrices. In the present research we evaluated 12 children with high functioning Autism and 6 with Asperger's syndrome through the WISC-IV and the Raven test. The results showed there are no significant differences between both measures of intelligence. However, we found that there are differences in the intellectual profile of both groups and that Asperger's syndrome appears to have a better intellectual level compared with high functioning Autism.

Key words: autism; Asperger's syndrome; intelligence; WISC; Raven.

\section{Resumo}

A inteligência nos transtornos do espectro autista, especialmente no autismo de alto funcionamento e a síndrome de Asperger, tem sido tema de numerosas pesquisas que pretendam estabelecer a capacidade intelectual que se observa nestes sujeitos. Alguns dos resultados mais sobressalientes assinalam que a inteligência fluida é um dos aspectos de desempenho superior nesta população, em comparação com o desempenho nas provas que medem inteligência cristalizada como as escalas Wechsler, razão pela qual os estudos sobre inteligência deveriam utilizar instrumentos que meçam o razoamento abstrato e a capacidade analógica, funções estreitamente relacionadas com a inteligência fluida, como é o teste de matrizes progressivas de Raven. Na presente pesquisa avaliaram-se 12 crianças com autismo de alto funcionamento e 6 com síndrome de Asperger, através do WISC-IV e o teste de Raven. Os resultados mostraram que não existem diferenças significativas entre ambas as medidas de inteligência. Não obstante, sim encontrou-se que existem diferenças no perfil intelectual de ambos os grupos e que a síndrome de Asperger parece ter um nível intelectual superior, em comparação com o autismo de alto funcionamento.

Palavras chave: autismo; síndrome de Asperger; inteligência; WISC; Raven.
La inteligencia es un proceso psicológico superior que abarca de manera global una serie de dominios intelectuales que le permiten al sujeto llevar a cabo actividades de razonamiento y solución de problemas, lo que facilita su adaptación exitosa al ambiente y responder de forma adecuada a las demandas y exigencias que este le presenta (Gardner, 2001; Nader \& Benaín, 2006). El estudio de la inteligencia en los trastornos del espectro autista (TEA) ha sido de interés desde hace más de medio siglo, como respuesta ante la asociación que se estableció entre estos trastornos y el retraso mental (RM) (Goldberg, 2006; López, Rivas \& Taboada, 2009). En referencia a esto último, los manuales de semiología diagnóstica - Manual diagnóstico y estadístico de los trastornos mentales (DSM-IV), de la American Psychiatric Association (2002), y la Clasificación internacional de enfermedades (CIE-10), de la Organización Mundial de la Salud (2002) — sugieren que si bien la presencia de RM no es determinante en el diagnóstico, es posible observar comorbilidad, exceptuando el síndrome de Asperger (SA), en el cual no debe existir déficit cognitivo.

En las últimas revisiones de los manuales diagnósticos como el DSM-V (American Psychiatric Association, 2014) desaparecen "los trastornos del espectro autista”, como el síndrome de Rett y el de Asperger, y queda una categorización por niveles de funcionalidad en los que se requiere indicar la gravedad de los síntomas conductuales y de la comunicación; así mismo, se ofrecen criterios para especificar si el trastorno se observa en compañía o en ausencia de deterioro intelectual, y así la presencia del déficit cognitivo corresponde a los niveles de funcionalidad más bajos.

De acuerdo con Ardila y Rosselli (2007), en los TEA se producen alteraciones cognoscitivas que pueden confundirse con el RM; la diferencia está en que el perfil del niño autista en pruebas psicométricas de inteligencia muestra una disociación entre el coeficiente intelectual verbal y el no verbal, donde las habilidades no verbales son muy superiores a las verbales, lo que no ocurre en niños con RM. En 
esta misma línea, Dawson, Soulières, Gernsbancher y Mottron (2007), en un estudio sobre la capacidad intelectual en población autista, aplicando la Escala de Inteligencia de Wechsler para niños (WISC-IV) y el Test de Matrices Progresivas de Raven, encontraron que los sujetos con este trastorno obtenían un rendimiento superior en esta última prueba (se ubicaban sobre el percentil 70). Este hallazgo fue confirmado más adelante en otras investigaciones realizadas, las cuales concluyen que el alto desempeño de los niños con autismo, en este tipo de tareas, está relacionado con la capacidad superior que tienen para procesar estímulos no verbales, y con un posible nivel superior de inteligencia fluida (Chen, Planche \& Lemonnier, 2009; Merchán-Naranjo et al., 2011).

Sin embargo, se ha observado que estas discrepancias no son iguales en todos los subtipos de autismo; por ejemplo, en una investigación realizada con sujetos con SA, se encontró que el desempeño era superior en la escala verbal que en la manipulativa en la escala de Wechsler. Este alto rendimiento en las tareas verbales encontrado en personas con SA se ha correlacionado de forma positiva con un lenguaje conversacional adecuado, presencia de relaciones sociales correctas, con coeficientes intelectuales elevados o normales (Sattler \& Hoge, 2008) y con un buen rendimiento en el Test de Raven, por lo cual se sugiere la posibilidad de que en estos individuos exista un nivel superior de inteligencia fluida (Hayashi, Kato, Igarashi \& Kashima, 2008). En relación con estos resultados, y utilizando la prueba de inteligencia Standford-Binet, Lennen, Lamb, Dunagan y Hall (2010) encontraron en una muestra de autistas una discrepancia entre el perfil verbal y el no verbal. Los resultados también sugirieron que existe déficit importante en las tareas que requerían el uso de la memoria de trabajo y en conocimiento (vocabulario), pero que había fortalezas en las actividades de razonamiento cuantitativo.

Resultados de otras investigaciones que han utilizado el WISC-IV han mostrado que los indi- viduos con SA obtienen las mejores puntuaciones en la prueba de información, en la que se requiere el almacenamiento de información general, al igual que vocabulario; también se observan buenos puntajes en semejanzas, en la cual es necesario el uso de la capacidad de razonamiento verbal, habilidad que se encuentra conservada (Pennington \& Ozonoff, 1996) y en la prueba de cubos, posiblemente a que su capacidad para fijarse en los detalles les representa una ventaja en la resolución de esta tarea (McArthur \& Adamson, 1996). Sin embargo, suelen mostrar puntuaciones más bajas en la tarea de rompecabezas, que demanda una buena capacidad de integración visomotora, la cual está afectada en este trastorno (Ozonoff, Pennington \& Rogers, 1996); en comprensión, debido a su clara relación con el juicio social (Ayuda-Pascual \& Martos-Pérez, 2007), y en claves, debido a las dificultades atencionales y al extremo perfeccionismo que dificulta la ejecución de la prueba (Equipo Deletrea \& Artigas, 2004).

Estudios relacionados con estos resultados han señalado que en las personas con SA existen falencias en el componente pragmático del lenguaje (Etchepareborda, 2001), dificultades ejecutivas asociadas con el déficit en el razonamiento verbal, la memoria verbal, el lenguaje complejo y la flexibilidad cognitiva (Ochoa \& Cruz, 2007), así como deficiencias en la comunicación entre los sistemas de memoria episódica y semántica (Margulis, 2009).

Actualmente, los estudios sobre inteligencia en los TEA han estado relacionados con el grado de funcionalidad de los pacientes y han generado expectativas sobre un nivel intelectual normal e, incluso, superior en diagnósticos de autismo de alto funcionamiento (AAF) y de SA. De este modo, se cree que en el SA no existe un retraso clínicamente significativo del desarrollo cognitivo, pero sí existen islotes de capacidad de ciertas áreas específicas, como la memoria semántica (Calle de Medinaceli \& Utria, 2004). En esta misma línea, Artigas (2000) considera que en el SA 
la inteligencia se encuentra dentro del rango de normalidad, y que aun las personas con este diagnóstico pueden tener determinadas habilidades cognitivas, excepcionalmente desarrolladas. Pero lo más común es que posean un cociente intelectual total normal-medio o normal-bajo. Otros enfoques han propuesto que si bien no existe un retardo mental en el autismo, sí existen perfiles intelectuales que permiten diferenciar un SA o un AAF de un autismo profundo (Dawson et al., 2007). Incluso algunos autores consideran la posibilidad de que la evaluación de las funciones cognitivas permitiría discriminar entre los autistas con RM y los autistas sin RM, así como diferenciar las personas con TEA de los individuos con desarrollo normal (Lennen et al., 2010). De lo anterior se entiende que en los TEA se encuentran afectados ciertos dominios cognitivos, a partir de los cuales es posible plantear hipótesis que busquen explicar la manifestación de los déficits, que a su vez permitan comprender la variabilidad de síntomas presentes en cada uno de los diferentes subtipos, incluidos los aspectos neuropsicológicos.

A pesar de la gran variabilidad en la sintomatología y etiología de los TEA, todos se caracterizan por la manifestación de las conductas nucleares descritas en los manuales de semiología clínica. No obstante, la consideración de los subtipos está determinada por otros factores; uno de ellos es sin duda el nivel intelectual. Un cociente intelectual por debajo de 70, combinado con un retraso en el desarrollo de las habilidades lingüísticas y mayores dificultades en el comportamiento adaptativo, se encuentra asociado con etiologías biológicas y genéticas y, por lo general, corresponden a diagnósticos clínicos de bajo funcionamiento como el síndrome de Rett, el trastorno desintegrativo infantil y el trastorno autista de bajo funcionamiento. Por otra parte, la presencia de capacidad intelectual normal, habilidades lingüísticas relativamente conservadas, mejor comportamiento adaptativo y ausencia de daño cerebral o etiología biológica se asocian con trastornos de alto funcionamiento como el SA, el AAF y el TEA no especificado (Rapin, 1999; Rinehart, Bradshaw, Brereton \& Tonge, 2002). En cuanto a las diferencias específicas entre el SA y el AAF, se ha observado que en el AAF se exhiben mayores índices de desviación en la adquisición del lenguaje; mientras que el SA se caracteriza por un discurso más complejo, pero acompañado de un tono pedante y la presencia de monólogos que incluyen un vocabulario elevado y sofisticado. También se cree que la variabilidad en el desempeño en la escala de Wechsler, entre el SA y el AAF, es un elemento que se debe considerar en el momento de establecer los límites entre ambos trastornos (Roselli, Matute \& Ardila, 2010).

La construcción del perfil intelectual de los TEA de alto funcionamiento (SAy AAF), que se muestra en el presente trabajo, aunque basada en evidencia empírica, no puede contrastarse con gran cantidad de estudios, ya que los reportados en la literatura son pocos (Dawson et al., 2007; Merchán-Naranjo et al., 2011); además, los hallazgos son contradictorios en algunas de estas investigaciones (Lennen et al., 2010 Williams et al., 2008), por lo cual se requiere la elaboración de protocolos de evaluación que incluyan pruebas válidas para las características que presenta la población, y que permitan la descripción adecuada de la capacidad cognitiva de los sujetos. Por esta razón, es evidente que son necesarios estudios que tengan por objetivo elucidar la relación entre los trastornos del espectro autista y el retardo mental, y que permitan obtener información para construir los perfiles intelectuales de los sujetos con TEA, y también investigaciones que se aproximen a proponer cuáles instrumentos de evaluación de la inteligencia son adecuados para la población autista, dadas sus características cognitivas particulares. Finalmente, la elaboración de perfiles neurocognitivos, que den cuenta del funcionamiento de los procesos mentales de los sujetos, en el que se incluyan tanto las falencias como las fortalezas, es necesaria para el diseño de procesos de intervención que respondan a las necesidades particulares de la población con este 
tipo de trastornos (Mulas, Hernández-Muela, Etchepareborda \& Abad-Mas, 2004).

\section{Método}

\section{Participantes}

Dieciocho niños de instituciones especializadas de Bucaramanga (Colombia) y su Área Metropolitana, con edades entre 6 y 16 años, y con diagnóstico de SA y AAF, según la clasificación del DSM-IV-TR. Para su selección se tuvieron en cuenta los siguientes criterios de inclusión: cumplir con los criterios para SA según el DSM-IV-TR y el Cuestionario de Screening para Espectro Autista de Alto Funcionamiento; además de la firma del consentimiento informado por parte de los padres o representantes legales de los niños. Así mismo, se designaron los criterios de exclusión dentro de los cuales se encontraba: presentar trastornos o condiciones neurológicas diferentes al SA o al AAF.

\section{Consideraciones éticas}

Todos los sujetos participaron en la investigación de forma voluntaria, de acuerdo con los criterios éticos de participación informada para la investigación con humanos establecida por el Ministerio de Salud (Resolución 8430 del 4 de octubre de 1993). Adicionalmente, según esta misma resolución, el estudio fue identificado como de riesgo mínimo, dado que los participantes no corrieron ningún tipo de riesgo médico, ni se realizó intervención clínica o procedimientos invasivos. Después de ser debidamente comunicados de los procedimientos, los padres o los representantes de los niños firmaron un consentimiento informado escrito para autorizar la participación en la investigación (anexo 1). En dicho consentimiento se informó que cualquier voluntario podía retirarse del estudio en el momento en que lo deseara sin dar justificaciones y sin ser presionado a perma- necer en este; además de explicarse los objetivos, riesgos, beneficios y confidencialidad del estudio.

Los resultados generales no fueron publicados ni difundidos con los nombres de los participantes. Adicionalmente, los resultados obtenidos con la experiencia individual fueron trasmitidos, mediante un informe de desempeño escrito y verbal, a todos los sujetos que así lo solicitaron.

Teniendo en cuenta el principio del Código Ético correspondiente a la competencia del evaluador, previamente a la ejecución de esta investigación se realizó una capacitación, por parte del grupo investigador, para la adecuada aplicación de los instrumentos que formaron parte del protocolo de evaluación y de intervención.

\section{Instrumentos}

Ficha de ingreso. Es un formato en el que se registran los antecedentes médicos y psicológicos de los niños evaluados, a fin de explorar variables que puedan interferir con el desempeño en las pruebas que se van a aplicar. Estos datos se tuvieron en cuenta para la calificación e interpretación de los resultados obtenidos en los instrumentos de evaluación.

Cuestionario para Diagnóstico de Síndrome de Asperger (Ortiz, 2003). Es un instrumento diseñado para facilitar el diagnóstico del SA, teniendo como criterios de evaluación los síntomas y signos señalados en el DSM-IV.

Cuestionario de Screening para Espectro Autista de Alto Funcionamiento (Ehlers, Gillberg \& Wing, 1999). Este cuestionario evalúa los síntomas característicos del trastorno autista de alto funcionamiento en niños y adolescentes, teniendo en cuenta los criterios descritos en la literatura y en los manuales de clasificación diagnóstica. Dentro de la prueba se examinan las siguientes áreas: interacción social, problemas de comunicación, conductas restrictivas y repetitivas, torpeza motora 
y diferentes tipos de tics posibles o movimientos repetitivos o estereotipados.

Test de Matrices Progresivas de Raven (Escala Especial-para edades de 4 a 11 años) (Raven, 1984). Fue creado por J. C. Raven, en 1938, para medir la capacidad intelectual mediante procesos de comparación de formas y de razonamiento analógico, con independencia de los conocimientos adquiridos, específicamente mide el factor $g$ de inteligencia, el cual es considerado la capacidad general de razonamiento que pone en marcha una serie de procesos cognitivos superiores que hacen apta a la persona para resolver problemas. La Escala Especial es una versión modificada para evaluar dicha capacidad en niños y personas intelectualmente discapacitadas.

Escala de Inteligencia de Wechsler para Niños (Wechsler, 2005). Es una escala que tiene como objetivo evaluar las capacidades cognitivas de niños y adolescentes con edades comprendidas entre los 6 y 16 años de edad. A partir de su aplicación se puede obtener una aproximación al funcionamiento cognitivo e intelectual del individuo. La prueba está conformada por cuatro índices: compresión verbal $(\mathrm{CV})$, razonamiento perceptivo (RP), memoria de trabajo (MT) y velocidad de procesamiento (VP), evaluados a través de quince tests, diez principales: cubos, semejanzas, dígitos, conceptos, claves, vocabulario, letras y números, matrices, comprensión y búsqueda de símbolos, y cinco optativos: figuras incompletas, animales, información, aritmética y adivinanzas. A partir de la suma de los cuatro índices anteriores se obtiene el cociente intelectual total que ubica al sujeto en un rango de desempeño respecto a su grupo poblacional de referencia.

\section{Procedimiento}

En primer lugar, se realizó la gestión y el contacto con las instituciones especializadas; a continuación tuvo lugar la selección de la muestra y la firma de consentimiento informado y el diligenciamiento de la ficha de ingreso. La aplicación de pruebas tuvo lugar en tres sesiones para cada participante, con una duración aproximada de sesenta minutos. Durante la primera se administró las escalas comprensión verbal y razonamiento perceptual del WISC-IV; en la segunda, los índices memoria de trabajo y velocidad de procesamiento, $y$, finalmente, el Test de Matrices Progresivas de Raven.

\section{Análisis de resultados}

Los resultados de las pruebas aplicadas se han organizado en datos descriptivos y datos comparativos. Dentro de los datos descriptivos se incluyen los promedios del Test de Matrices Progresivas de Raven y el WISC-IV, tanto en los puntajes directos como en las puntuaciones estándar. Por otra parte, en los datos comparativos se incluyen los análisis de comparación de medias (T de Student) de las pruebas realizadas, teniendo como criterio de contraste dos grupos: SA y AAF. Los análisis se realizaron también con puntajes directos y con puntajes corregidos. También se realizó una correlación de Pearson entre los puntajes directos obtenidos en el WISC-IV y el Raven, así como en los puntajes corregidos. Para todos los análisis se designó un nivel de significancia de $.050(p<.05)$.

\section{Resultados}

\section{Descripción sociodemográfica de la muestra}

Participaron en la investigación un total de 18 niños, de los cuales 12 contaban con un diagnóstico de AAF, y 6, con un dictamen de SA. La edad promedio de los participantes para el grupo de AAF fue de 7 años 4 meses; mientras que para el de SA fue de 6 años, 9 meses. La distribución de hombres y mujeres fue desigual en ambos grupos, siendo mayor el número de participantes hombres. En el grupo de AAF había 2 casos femeninos y en el de SA un solo caso (tabla 1). 
Tabla 1

Variables sociodemográficas (edad y sexo) de los participantes de los grupos evaluados: autismo de alto funcionamiento y sindrome de Asperger

\begin{tabular}{lccc}
\hline Variable & $\begin{array}{c}\text { Autismo de alto } \\
\text { funcionamiento }\end{array}$ & $\begin{array}{c}\text { Síndrome de } \\
\text { Asperger }\end{array}$ & $\begin{array}{c}\text { Total } \\
\text { población }\end{array}$ \\
\hline $\begin{array}{l}\text { Edad pro- } \\
\text { medio }\end{array}$ & 7.4 & 6.9 & 7.3 \\
Mujeres & 2.0 & 1.0 & 3.0 \\
Hombres & 10.0 & 5.0 & 15.0 \\
\hline
\end{tabular}

Datos descriptivos de los puntajes directos de la muestra en las pruebas de inteligencia (WISC-IV y Test de Matrices Progresivas de Raven)

A continuación se observan las medias de los puntajes obtenidos por los participantes en cada una de las subpruebas del WISC-IV y en el Raven. También se incluye el número de sujetos que contestaron cada test. Las subpruebas Información y Adivinanzas no se aplicaron a ningún participante, en razón a que los sujetos se veían imposibilitados a contestar este tipo de tareas, debido a su naturaleza verbal, por lo cual no hay datos de estas. La prueba del WISC-IV en la cual se obtuvo mejor desempeño fue en el subtest de Aritmética (18.3) y la de peor rendimiento fue Comprensión (4.7) (tabla 2).
Datos descriptivos de los puntajes corregidos de la muestra en las pruebas de inteligencia (WISC-IV y Test de Matrices Progresivas de Raven)

En los puntajes corregidos, en el WISC-IV el puntaje mayor se obtuvo en el índice de Razonamiento Perceptual (78.9), y el de Comprensión Verbal (78.9), los cuales se ubican en el grupo de comparación como un puntaje normal-bajo, y el de menor rendimiento fue Memoria de Trabajo (67.7), siendo esta una puntuación muy baja en comparación con el grupo de referencia. Los demás puntajes también se ubican en un rango inferior para lo esperado, según el grupo de edad (figura 1). En cuanto al Raven, la puntuación percentil corresponde a una interpretación cualitativa de inferioridad intelectual (figura 2).

\section{Datos comparativos de los desempeños en el WISC-IV y el Raven entre los grupos de AAF y SA}

En los puntajes directos se compararon los subtest del WISC-IV en ambos grupos, así como los resultados del Raven. Se encontraron diferencias estadísticamente significativas en las pruebas de Semejanzas ( $p=.016)$, Comprensión $(p=.013)$ y Dígitos $(p=.038)$. Nótese también que los promedios indican que el grupo de SA alcanzó un mayor desempeño en comparación con el de AAF en estas mismas pruebas (tabla 3).

Tabla 2

Promedios de los puntajes directos en las pruebas aplicadas en el Raven y el WISC-IV

\begin{tabular}{|c|c|c|c|c|c|c|c|c|c|c|c|c|}
\hline \multicolumn{13}{|c|}{ Resultados promedio de las subpruebas aplicadas (puntuación directa) } \\
\hline & \multicolumn{11}{|c|}{ WISC-IV } & \multirow[t]{2}{*}{ Raver } \\
\hline & $\mathrm{CC}$ & $\mathrm{S}$ & $\mathrm{D}$ & Co & $\mathrm{Cl}$ & $\mathrm{V}$ & M & $\mathrm{C}$ & BS & FI & A & \\
\hline$N$ & 10.0 & 10.0 & 9.0 & 7.0 & 12.0 & 10.0 & 12.0 & 10.0 & 11.0 & 11.0 & 11.0 & 14.0 \\
\hline Promedio & 15.2 & 10.5 & 6.7 & 5.7 & 25.5 & 11.8 & 8.11 & $4.7^{*}$ & 13.5 & 16.8 & $18.3^{* *}$ & 12.7 \\
\hline
\end{tabular}

*Puntuación más baja; **puntuación más alta.

CC: Cubos; S: Semejanzas; D: Dígitos; Co: Conceptos; Cl: Claves; V: Vocabulario; M: Matrices; C: Comprensión; BS: Búsqueda de Símbolos; FI: Figuras Incompletas; A: Aritmética. 


\section{Paula fernanda Pérez Rivero, Lía Margarita Martínez Garrido}

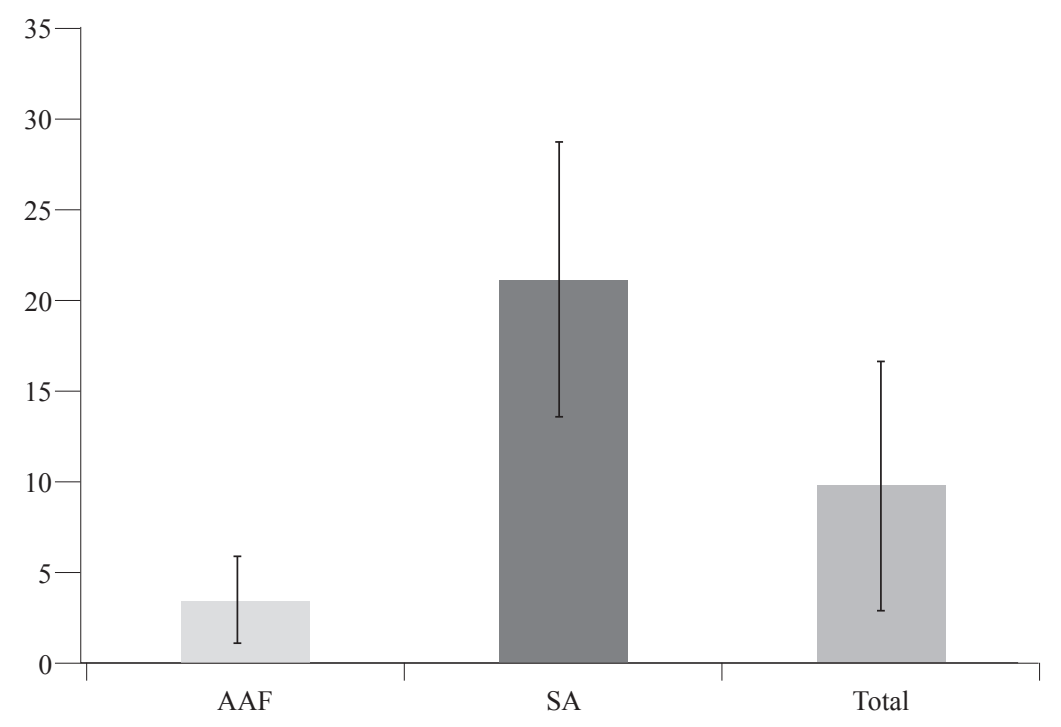

Figura 1. Promedio del desempeño (EEM) de los grupos con SA y AAF en el WISC-IV (puntajes corregidos)

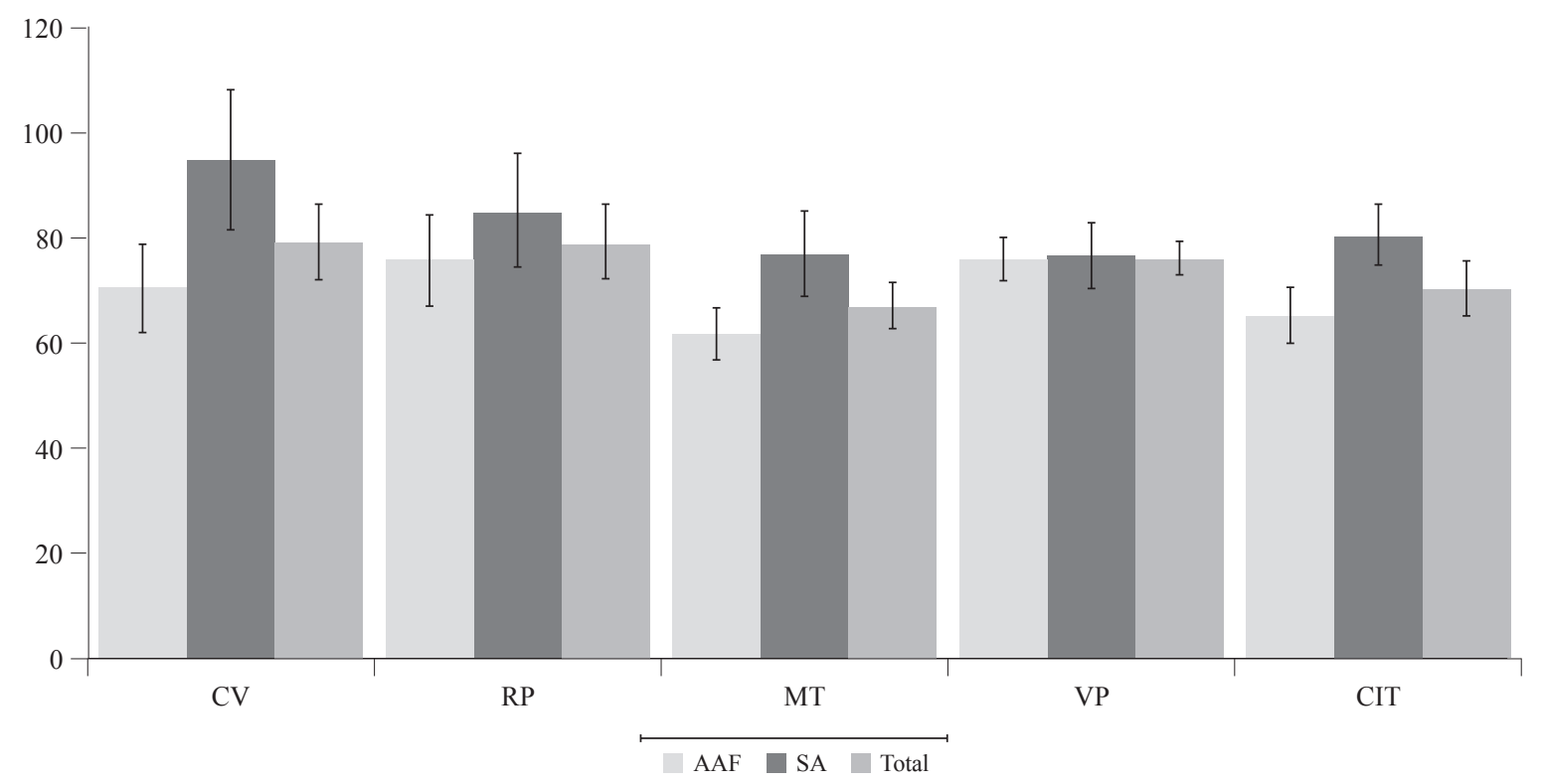

Figura 2. Promedio del desempeño (EEM) de los grupos con SA y AAF en el Raven (puntajes corregidos)

CV: Compresión Verbal; RP: Razonamiento Perceptual; MT: Memoria de Trabajo; CIT: Coeficiente Intelectual Total. 
Tabla 3

Comparación de medias entre el SA y el AAF (puntajes directos)

\begin{tabular}{lrrr}
\hline \multicolumn{1}{c}{$T$ de Student para muestras independientes SA vs. AFF } \\
\cline { 1 - 3 } \multicolumn{1}{c}{ Pruebas } & \multicolumn{3}{c}{ Media } \\
\cline { 2 - 3 } & \multicolumn{1}{c}{ SA } & \multicolumn{1}{c}{ AFF } \\
\cline { 2 - 3 } Cubos (CC) & 12.0 & 13.54 & .784 \\
Semejanzas (S) & 17.6 & 8.2 & $.016^{*}$ \\
Dígitos (D) & 10.0 & 6.0 & $.038^{*}$ \\
Conceptos (Co) & 6.3 & 6.1 & .952 \\
Claves (Cl) & 25.0 & 26.4 & .862 \\
Vocabulario (V) & 23.0 & 15.0 & .154 \\
Matrices (M) & 7.0 & 5.5 & .886 \\
Comprensión (C) & 8.0 & 4.3 & $.013^{*}$ \\
Búsqueda de Símbolos (BS) & 15.0 & 15.0 & .947 \\
Figuras Incompletas (FI) & 14.0 & 14.0 & .943 \\
Aritmética (A) & 4.0 & 7.1 & .137 \\
Raven & 18.83 & 15.91 & .340 \\
\hline
\end{tabular}

*Diferencia estadísticamente significativa $(p<.05)$.

En cuanto a los puntajes corregidos, la comparación entre medias arrojó la existencia de diferencias estadísticamente significativas, entre ambos grupos, para el índice de Comprensión Verbal $(p=.023)$ del WISC-IV (tabla 4).

Tabla 4

Comparación de medias entre el SA y el AFF (puntajes corregidos)

\begin{tabular}{cccc}
\hline \multicolumn{4}{c}{$T$ de Student para muestras independientes } \\
\cline { 2 - 3 } Variable & \multicolumn{3}{c}{ Media } \\
\cline { 2 - 3 } & 5.00 & 5.00 & .609 \\
\hline Raven & 94.83 & 71.25 & $.023^{*}$ \\
CV & 84.83 & 79.00 & .897 \\
RP & 71.50 & 57.50 & .140 \\
MT & 76.33 & 76.50 & .688 \\
VP & 80.33 & 67.25 & .255 \\
CIT &
\end{tabular}

*Diferencia estadísticamente significativa $(p<.05)$.

CV: Compresión Verbal; RP: Razonamiento Perceptual; MT: Memoria de Trabajo; VP: Velocidad de Procesamiento; CIT: Coeficiente Intelectual Total.
Adicionalmente, se realizó una $T$ de Student para comparar los desempeños por género, pero no se encontraron diferencias estadísticamente significativas. También se ejecutó un análisis de varianza (Anova) de una vía, para comparar los resultados entre los diferentes grupos de edad y se encontró la no existencia de diferencias estadísticamente significativas. Finalmente, en los análisis de comparación de medias no se hallaron diferencias estadísticamente significativas entre los subtest del WISC-IV y el puntaje del Raven.

\section{Correlación entre los desempeños de los índices del WISC-IV y los desempeños en el Test de Matrices Progresivas de Raven en los grupos de AAF y SA}

Se encontraron relaciones positivas estadísticamente significativas entre el desempeño en los siguientes índices y el Raven: Memoria de Trabajo $(r=.729 ; p=.000593)$, Razonamiento Perceptual $(r=.582 ; p=.0113)$ y coeficiente intelectual total $(r=.625 ; p=.00558)$. En los otros índices no se hallaron relaciones estadísticamente significativas (tabla 5).

Tabla 5

Correlación entre los índices del WISC-IV y el Raven

\begin{tabular}{|c|c|c|c|c|c|c|c|}
\hline \multicolumn{2}{|c|}{ Variables } & Raven & $\mathrm{CV}$ & $\mathrm{RP}$ & MT & VP & CIT \\
\hline 1 & Raven & & & & & & \\
\hline 2 & $\mathrm{CV}$ & $\begin{array}{l}.429 \\
.0758\end{array}$ & & & & & \\
\hline 3 & $\mathrm{RP}$ & $\begin{array}{l}.582^{*} \\
.0113\end{array}$ & $\begin{array}{l}.503^{*} \\
.033\end{array}$ & & & & \\
\hline 4 & MT & $\begin{array}{c}.729^{*} \\
.000593\end{array}$ & $\begin{array}{l}.713^{*} \\
.0009\end{array}$ & $\begin{array}{c}.838^{*} \\
.00001\end{array}$ & & & \\
\hline 5 & VP & $\begin{array}{l}.352 \\
.152\end{array}$ & $\begin{array}{l}.351 \\
.153\end{array}$ & $\begin{array}{l}.776^{*} \\
.0001\end{array}$ & $\begin{array}{l}.519^{*} \\
.0275\end{array}$ & & \\
\hline 6 & CIT & $\begin{array}{l}.625^{*} \\
.00558\end{array}$ & $\begin{array}{l}.766^{*} \\
.0002\end{array}$ & $\begin{array}{c}.932^{*} \\
.00000001\end{array}$ & $\begin{array}{c}.915^{*} \\
.0000001\end{array}$ & $\begin{array}{c}.749^{*} \\
.000351\end{array}$ & \\
\hline
\end{tabular}

*Relación estadísticamente significativa $(p<.05)$.

CV: Compresión Verbal; RP: Razonamiento Perceptual; MT: Memoria de Trabajo; VP: Velocidad de Procesamiento; CIT: Coeficiente Intelectual Total. 


\section{Discusión}

La evaluación de la inteligencia en la población autista es actualmente un reto para la neuropsicología. A pesar de los estudios que se han realizado, no ha sido posible llegar a un consenso sobre la mejor manera de establecer el estatus intelectual de las personas diagnosticadas con este tipo de trastornos. De hecho, algunos autores proponen que los síntomas del autismo tienen influencia en el proceso de evaluación. Así, se cree que los déficits de lenguaje, atención y motivación, que están a menudo presentes en los autistas, pueden afectar los resultados de la valoración intelectual (Goldberg, 2004).

Los intentos por aproximarse a este fenómeno han llevado a diferentes hallazgos y posturas; en un primer momento se pensó que de forma general existía un déficit cognitivo que podría asemejarse a un retardo mental (Asperger, 1979; Wing, 1981) y que estaba relacionado de forma muy estrecha con deficiencias del lenguaje y la comunicación (Campos, 2007). Sin embargo, otras observaciones llevaron a la conclusión de que en el RM existe una alteración global en todas las funciones cognitivas; por el contrario, en el autismo, especialmente en el SA y el AFF, existen deficiencias en algunas funciones, en tanto que en otras se evidencia una capacidad sobresaliente (Ardila \& Roselli, 2007). Algunos autores consideran que esta capacidad sobresaliente se constituye en una forma de compensar las alteraciones que existen en las otras funciones (Calle de Medinaceli \& Utria, 2004), mientras que otros consideran que esta es una posición que subvalora las capacidades de los autistas y que deberían considerarse las habilidades extraordinarias como una manifestación de la inteligencia de estos individuos (Bölte \& Poustka, 2004).

No obstante, más allá de si las capacidades de los autistas son consideradas parte del trastorno o como habilidades preservadas, se debe tener en cuenta que la oportuna evaluación del estado intelectual en esta población es importante para el diseño de programas de rehabilitación (Portellano, 2008), además de que favorece el proceso de diagnóstico, puesto que si bien aún existe la discusión sobre la comorbilidad del RM y el autismo (Goldberg, 2006), varios estudios coinciden en que hay un perfil intelectual particular en estos trastornos (Artigas, 2000; Chen et al., 2010; Etchepareborda, 2001) que los diferencia entre sí, así como de otros cuadros similares. Esto último coincide con lo encontrado en los análisis de comparación de medias ( $T$ de Student), realizados en el presente trabajo entre el SA y el AAF, en las pruebas de inteligencia (WISC-IV y Raven), los cuales arrojaron que existen diferencias estadísticamente significativas en el desempeño de las subpruebas de Dígitos, Semejanzas y Comprensión, y de forma general en el índice de Comprensión Verbal.

Otro punto de discusión consiste en los instrumentos utilizados para la medida de la capacidad intelectual en este grupo de personas. Por ejemplo, el uso de las escalas Wechsler ha sido arduamente criticado en razón a que en ellas existe un componente verbal, dimensión que está afectada en los TEA (Muñoz \& Martínez, 2008). En esta misma línea, Lennen et al. (2010), al evaluar las propiedades psicométricas del test, encontraron que las habilidades verbales son determinantes a la hora de solucionar las tareas que este propone, incluso en aquellos subtest que no tienen, aparentemente, un componente verbal. Por esta razón se ha sugerido que la evaluación de la inteligencia sea realizada a través de pruebas no verbales, como el Test de Matrices Progresivas de Raven (Dawson et al., 2007), para asegurar que la naturaleza de la prueba no condicione los resultados obtenidos. No obstante, la aplicación del WISC-IV, en esta población, ha permitido hacer una descripción más completa de las funciones cognitivas, porque evalúa varias dimensiones, en lugar de solo una, como ocurre con el Raven.

En esta misma línea, la discrepancia que reporta la literatura en el desempeño en la escala de Wechsler en población autista (Artigas, 2000; 
Bölte, Dziobek \& Poustka, 2009; Hayashi et al., 2008; Merchán-Naranjo et al., 2011) fue observada en los resultados obtenidos en la presente investigación, donde el rendimiento en el índice de comprensión verbal fue superior en el SA que en el AAF, así como en las subpruebas de Semejanzas, Comprensión y Vocabulario que componen el índice, mientras que en los niños con AAF se observó un mejor desempeño en tareas no verbales, como Claves, Búsqueda de Símbolos, Matrices y Cubos, que están relacionadas con el razonamiento perceptual. No obstante, el desempeño en el Raven fue mejor en el SA que en el AAF, lo cual contradice la hipótesis de que en el SA existe una mayor inteligencia cristalizada; mientras que en el AAF, una mayor inteligencia fluida (Merchán-Naranjo et al., 2011).

Cabe añadir al respecto que el desempeño en el WISC-IV también fue superior en el SA, por lo que se podría afirmar que, independientemente de la medida de inteligencia que se obtenga (cristalizada o fluida), las personas con SA tienen un perfil intelectual superior, en comparación con los sujetos con AAF. Una hipótesis que podría explicar este hecho es que es posible que en algunos de los participantes evaluados existiera un cuadro comórbido de RM, a pesar de ser más funcionales en su comportamiento adaptativo y tener mejores habilidades lingüísticas, en comparación con los individuos con autismo de bajo funcionamiento (Bölte et al., 2009). Otra postura teórica que podría explicar los resultados obtenidos es la que propone que en el autismo existe un continuo de funcionalidad (entendida esta en tres dimensiones: nivel adaptativo, nivel comunicativo y nivel intelectual), en el cual el SA corresponde al trastorno de mejor pronóstico, mejor estatus cognitivo, presencia de habilidades lingüísticas avanzadas y mejor comportamiento adaptativo; seguido del AAF, en el que se observan algunos déficits en los niveles mencionados, $\mathrm{y}$, finalmente, el autismo clásico o de bajo funcionamiento, en el cual estas habilidades están profundamente afectadas, además de que generalmente existe un diagnóstico de RM adicional (Campos, 2007; Mendoza \& Muñoz, 2005).

Cabe añadir en este punto que la interpretación cualitativa de los resultados del WISC-IV ubica los niños con AAF como inferiores a lo esperado para el grupo de referencia; mientras que los puntajes en el SA se corresponden con diagnósticos de rendimiento intelectual promedio y normal-bajo. Este resultado coincide con lo que sugiere la literatura, según la cual el rendimiento intelectual general en el SA se encuentra dentro de los valores normales, y que además este rendimiento es superior, en comparación con el grupo de AAF (Artigas, 2000). En cuanto al Raven, todos los participantes se ubican en percentiles muy por debajo de la media esperada para su grupo de referencia; este resultado contrasta con lo encontrado en otras investigaciones que afirman que los TEA obtienen buen rendimiento en esta prueba (Dawson et al., 2007; Sattler \& Hoge, 2008), pero es coherente con otros hallazgos, según los cuales el rendimiento deficiente de los pacientes con autismo, para ejecutar tareas de razonamiento visoperceptual, puede ser entendido como una discapacidad no verbal de aprendizaje, la cual se debe a una afectación global del hemisferio derecho (Williams et al., 2008).

Por otra parte, el análisis de coeficiente de correlación de Pearson arrojó que existe una relación positiva estadísticamente significativa entre los índices de Memoria de Trabajo y Razonamiento Perceptual del WISC-IV y el Raven, así como también entre el coeficiente intelectual total y el Raven.

En cuanto a la relación Memoria de Trabajar-Raven, se podría afirmar que, en términos funcionales, existe entre el factor $g$ de inteligencia y la memoria de trabajo. Dicha relación ha sido encontrada en numerosos estudios (Ackerman, Beier \& Boyle, 2002; Colom \& Andrés-Pueyo, 2005; Colom, Rubio, Chun Shih \& Santacreu, 2006; Kyllonen \& Christal, 1990). Algunas explicaciones sobre la naturaleza de esta relación apuntan a que, debido a que la memoria de trabajo no está relacionada con estructuras conceptuales, es decir, 
es una función cognitiva que trabaja sobre otras funciones mentales independientemente del conjunto de conocimientos que son aplicadas a estas, estaría directamente en relación con el factor gene$\mathrm{ral}(g)$ de inteligencia que subyace a otros procesos mentales, y que de igual forma no depende de la cantidad de conocimientos acumulados (Oberauer, Schulze, Wilhelm \& Su“ $\beta$, 2005). Otros investigadores han sugerido que la relación se explica porque la memoria de trabajo constituye un sistema en el que los sujetos almacenan transitoriamente la información y la someten a procesamiento, lo que genera como resultado una respuesta acertada a una tarea dada (Colom \& Flores, 2001), por lo cual, al permitir un adecuado procesamiento de la información de entrada, se amplía la capacidad para resolver problemas, utilizando los recursos cognitivos necesarios, y se evidencia así un buen nivel de inteligencia fluida $(g)$.

En coherencia con lo anterior, los puntajes obtenidos en el índice de Memoria de Trabajo en la población evaluada fueron los que peor desempeño evidenciaron. Tal resultado estaría relacionado con el bajo desempeño en el test de Raven, que contradice nuevamente la hipótesis de que en la población autista existe un nivel superior de inteligencia fluida. Además, si la naturaleza de la relación entre Memoria de Trabajo y $g$ es la descrita en las investigaciones mencionadas, no sería posible que las personas con autismo tuvieran desempeños altos en Raven, en razón a que, de hecho, se han encontrado alteraciones en la memoria de trabajo en personas con este trastorno, especialmente en el grupo con AAF (Boucher \& Warrington, 1976; Romero-Munguía, 2002).

Ahora bien, la relación entre el Raven y el índice de Razonamiento Perceptual se justifica porque ambas pruebas apuntan a la medición de procesos de razonamiento abstracto, procesamiento gestáltico, razonamiento eductivo, capacidad visoperceptual y razonamiento espacial (Portellano, 2008; Raven, 1984). Además, conceptualmente se entiende que $g$ está relacionada con la complejidad de la actividad cognitiva exigida por los problemas, es decir, la capacidad para captar relaciones entre elementos, conceptos abstractos, razonar, analizar, hallar características comunes entre elementos superficialmente distintos, e inferir conclusiones a partir de los elementos de información (Colom \& Andrés-Pueyo, 2005). Así mismo, el índice de Razonamiento Perceptual se define como la medida de la habilidad para interpretar y organizar material percibido visualmente, y para generar y probar hipótesis relacionadas en la solución de problemas; también, se considera una medida del razonamiento fluido y perceptivo, del procesamiento espacial y de la integración visomotora (Wechsler, 2005). De este modo, puede verse que ambas pruebas pretenden establecer el desempeño de las mismas funciones mentales.

La correlación encontrada entre el cociente intelectual total y el Raven sugiere que ambos instrumentos apuntan a la medición de la inteligencia, sin importar cuál medida de inteligencia es la que mayor peso tenga dentro de sus propiedades psicométricas (fluida o cristalizada). Adicionalmente, se considera que de forma general todos los test que miden inteligencia ofrecen una medida de $g$, en mayor o menor proporción (el Raven tiene mayor peso, que el WISC-IV, de $g$ ), debido a que esta capacidad está presente en las tareas propuestas para medir cociente intelectual, las cuales están estrechamente relacionadas con funciones superiores de solución de problemas, razonamiento, creación de estrategias y capacidad de adaptación ante estímulos nuevos. Las operaciones mentales que este tipo de problemas exigen a la persona intentan capturar procesos importantes presentes en las situaciones cotidianas, por lo cual tanto el WISC-IV como el Raven tienen validez predictiva en cuanto a la medición de la capacidad intelectual general (Colom \& Andrés-Pueyo, 2005).

La ausencia de relación entre el Raven y el índice de Comprensión Verbal se explica porque, esencialmente, el Raven es un test no verbal, independiente de los aprendizajes formales y de los 
conceptos presentes en los sistemas de memoria de los evaluados, mientras que el índice de Comprensión Verbal fue diseñado precisamente para obtener una medida de estas habilidades. De acuerdo con Wechsler (2005), la Comprensión Verbal evalúa los conocimientos y entendimiento verbal obtenidos por educación formal e informal, y es un reflejo de las habilidades verbales que tienen las personas para hacer frente a situaciones novedosas que exijan el uso del lenguaje verbal. Este resultado también es coherente con la discrepancia observada en la población con SA, en la cual se observó un buen desempeño en la escala de Comprensión Verbal; mientras que un pobre desempeño en Razonamiento Perceptual y Raven. Lo anterior confirma la idea de que en este trastorno la inteligencia cristalizada es superior a la fluida, en comparación con la población normal (Roselli et al., 2010). Por otra parte, la ausencia de correlación entre el índice de velocidad de Procesamiento y el Raven indica que no existe relación entre las medidas de velocidad de procesamiento y memoria visual inmediata y la medida de razonamiento abstracto e inteligencia fluida que evalúa el Raven.

Los estudios neuropsicológicos en la población autista han revelado que existe en esta población una serie de alteraciones en algunos procesos, como la atención, la memoria y el lenguaje; sin embargo, y a pesar de que muchos de estos resultados son consistentes entre sí, es necesario hacer una revisión meticulosa del método a través del cual se obtienen dichos resultados, de modo que se pueda conocer si existe la posibilidad de que estos respondan al modelo del experimento o si realmente muestran el funcionamiento cognitivo de los TEA. En cuanto a la versatilidad, es claro que en los TEA existen diferentes perfiles de desempeño, más o menos diferenciables entre sí, que hacen difícil la generalización de los síntomas. Esta controversia es alimentada por los casos excepcionales, en los cuales se observan capacidades extraordinarias combinadas con picos de deficiencia en otras áreas (Mottron, Dawson, Bertone \& Wang, 2007).
Lo anterior ha repercutido en una serie de interrogantes que llevan a pensar que si bien el trastorno autista está definido por una serie de alteraciones comunicativas, sociales y cognitivas, los casos de compensación o alto rendimiento en tareas específicas aún no han podido ser satisfactoriamente explicados. Quizás los reportes que mayor polémica causan son los relacionados con los savants, quienes demuestran habilidades extraordinarias en la música, las matemáticas, el dibujo y, por supuesto, islotes de alta capacidad en tareas visoespaciales y de memoria. Algunas de las explicaciones mayormente aceptadas son las que proponen que el autismo está caracterizado por un estilo cognitivo enfocado en el procesamiento local de la información, en contraposición al procesamiento global, y que este estilo de procesamiento hace parte del fenotipo autista y que se transmite genéticamente (Happé, 1999).

\section{Conclusiones}

Existen diferencias entre los perfiles intelectuales de los individuos con SA y las personas con AAF. En el SA hay un déficit del razonamiento perceptual y fortalezas en el componente verbal, siempre que este no esté asociado con el componente pragmático del lenguaje; mientras que en el AAF ocurre lo contrario: el razonamiento verbal es deficiente, pero existe una facilidad para dar respuesta a tareas con estímulos no verbales. Sin embargo, el nivel intelectual del SA es mayor que el de los niños con AAF. Por otra parte, aunque los desempeños en las pruebas de inteligencia en general fueron deficientes para el grupo con AAF, no es posible afirmar que existe un RM en las personas evaluadas, porque, como se menciona en la literatura, existen deficiencias que son compensadas con capacidades normales o sobresalientes en otras áreas. En otras palabras, lo que se sugiere es que el perfil intelectual en los niños con SA y AAF es heterogéneo. Finalmente, la medida de la capacidad intelectual en los TEA debe estar apoyada por 
la aplicación de varias pruebas neuropsicológicas, así como de la observación en diferentes contextos donde se ponga en evidencia la capacidad del sujeto para razonar, establecer relaciones lógicas y dar solución a los problemas que le demanda el ambiente. Esto en razón a que si bien las pruebas de inteligencia ofrecen un panorama, aún es incierto si su aplicación en este tipo de población es válida.

\section{Referencias}

Ackerman, P., Beier, M. \& Boyle, M. (2002). Individual differences in working memory within a nomological network of cognitive and perceptual speed abilities. Journal of Experimental Psychology: General, 131, 567-589.

American Psyquiatric Association. (2002). Manual diagnóstico y estadístico de los trastornos mentales. Barcelona: Masson.

American Psychiatric Association. (2014). Guia de consulta de los criterios diagnósticos del DSM5. Washington D.C.: autor.

Ardila, A. \& Roselli, M. (2007). Neuropsicología clínica. México D.F.: Manual Moderno.

Artigas, J. (2000). Aspectos neurocognitivos del síndrome de Asperger. Revista de Neurología Clínica, 1, 34-44.

Asperger, H. (1979). Problems of infantile autism. Communication, 13, 45-52.

Ayuda-Pascual, R. \& Martos-Pérez, J. (2007). Influencia de la percepción social de las emociones en el lenguaje formal en niños con síndrome de Asperger o autismo de alto funcionamiento. Revista de Neurología, 44, 57-59.

Bölte, S. \& Poustka, F. (2004). Comparing the intelligence profiles of savant and nonsavant individuals with autistic disorder. Intelligence, 32, 121-131.

Bölte, S., Dziobek, I. \& Poustka, F. (2009). Brief report: The level and nature of autistic intelligence revisited. Journal of Autism and Developmental Disorders, 39(4), 678-682.
Boucher, J. \& Warrington, E. (1976). Memory deficits in early infantile autism: Some similarities to the amnesic syndrome. British Journal of Psychology, 67, 73-87.

Calle de Medinaceli, J. \& Utria, O. (2004). Trastorno de Asperger en adolescentes: revisión del concepto y estrategias para la integración escolar. Revista Latinoamericana de Psicología, 36, 517-530.

Campos, C. (2007). Trastornos del espectro autista. México D. F.: Manual Moderno.

Chen, F., Planche, P. \& Lemonnier, E. (2010). Superior nonverbal intelligence in children with high-functioning autism or Asperger's syndrome. Research in Autism Spectrum Disorders, 4(3), 457-460.

Colom, R. \& Flores, C. (2001). Inteligencia y memoria de trabajo: La relación entre factor $\mathrm{g}$, complejidad cognitiva y capacidad de procesamiento. Psicologia: Teoria e Pesquisa, 17, 37-47.

Colom, R. \& Andrés-Pueyo, A. (2005). El estudio de la inteligencia humana: recapitulación ante el cambio de milenio. Psicothema, 11, 453-476.

Colom, R., Rubio, V., Chun Shih, P. \& Santacreu, J. (2006). Fluid intelligence, working memory and executive functioning. Psicothema, 18, 816-821.

Dawson, M., Souliéres, I., Gernsbacher, M. \& Mottron, L. (2007). The level and nature of autistic intelligence. Psychological Science, 18(8), 657-662.

Ehlers, S., Gillberg, C. \& Wing, L. (1999). A Screening Questionnaire for Asperger Syndrome and Other High-Functioning Autism Spectrum Disorders in School Age Children. Journal of Autism and Developmental Disorders, 29(2), 129-141.

Equipo Deletrea \& Artigas, J. (2004). Un acercamiento al síndrome de Asperger: una guía teórica y práctica. Madrid: Asociación Asperger España. Etchepareborda, M. (2001). Perfiles neuro-cognitivos del espectro autista. Revista de Neurología Clínica, 2(1), 175-192. 
Gardner, H. (2001). Estructuras de la mente: la teoría de las inteligencias múltiples. Bogotá: Fondo de Cultura Económica.

Goldberg, M. (2004). A car goes in the garage like a can of peas goes in the refrigerator: Do deficits in real-world knowledge affect the assessment of intelligence in individuals with autism? $\mathrm{Fo}_{\mathrm{O}}$ cus on Autism and other Developmental Disabilities, 20(1), 2-9.

Goldberg, M. (2006). Are the majority of children with autism mentally retarded? A systematic evaluation of the data. Focus on Autism and Other Developmental Disabilities, 21, 66-83.

Happé, F. (1999). Autism: Cognitive deficit or cognitive style? Trends in Cognitive Sciences, 3(6), 216-222.

Hayashi, M., Kato, M., Igarashi, K. \& Kashima, H. (2008). Superior fluid intelligence in children with Asperger's disorder. Brain and Cognition, 66(3), 306-310.

Kyllonen, P. \& Christal, R. (1990). Reasoning ability is (little more than) working memory capacity? Intelligence, 14, 389-433.

Lennen, D., Lamb, G., Dunagan, B. \& Hall, T. (2010). Verbal prowess equals higher IQ: Implications for evaluating autism. Research in Autism Disorders, 4(1), 95-101.

López, S., Rivas, R. \& Taboada, E. (2009). Revisiones sobre el autismo. Revista Latinoamericana de Psicología, 41, 555-570.

Margulis, L. (2009). Funcionamiento de los sistemas de memoria en niños con trastorno autista y trastorno de Asperger. Revista Argentina de Neuropsicología, 13, 29-48.

McArthur, D. \& Adamson, L. (1996). Joint attention in preverbal children: Autism and developmental language disorder. Journal of Autism and Developmental Disorders, 26(5), 481-496.

Mendoza, E. \& Muñoz, J. (2005). Del trastorno especifico del lenguaje al autismo. Revista de Neurología, 41, 91-98.

Merchán-Naranjo, J., Mayoral, M., Rapado-Castro, M., Llorente, C., Boada, L., Arango, C. \& Pare- llada, M. (2011). Estimation of the intelligence quotient using Wechsler Intelligence Scales in children and adolescents with Asperger syndrome. Journal of Autism and Developmental Disorders, 42(1), 116-122. doi: 10.1007/ s10803-011-1219-8

Mottron L., Dawson, M., Bertone, A. \& Wang, L. (2007). Cognitive versatility in autism cannot be reduced to deficit. Cognitive Neuropsychology, 24(5), 578-580.

Mulas, F., Hernández-Muela, S., Etchepareborda, M. \& Abad-Mas, L. (2004). Bases clínicas neuropediátricas y patogénicas del trastorno del espectro autista. Revista de Neurología, 38(1), 9-14.

Muñoz, J. \& Martínez, L. (2008). La escala de inteligencia de Wechsler para niños-IV (WISC-IV) en un grupo de discapacitados intelectuales. Psicología Educativa, 14, 29-46.

Nader, M., \& Benaín, D. (2006). La inteligencia de los niños. Psicología, Cultura y Sociedad, 5, 27-40.

Oberauer, K., Schulze, R., Wilhelm, O. \& Su“ $\beta$, M. (2005). Working memory and intelligencetheir correlation and their relation: Comment on Ackerman, Beier, and Boyle. Psychological Bulletin, 131, 61-65.

Ochoa, S. \& Cruz, I. (2007). Wisconsin Card Sorting Test en el estudio del déficit de atención con hiperactividad, trastornos psiquiátricos, autismo y vejez. Universitas Pychological, 6(3), 637-648

Ortiz, T. (2003). Síndrome de Asperger: criterios y cuestionario para facilitar el diagnóstico. $R e$ vista Neurología, Neurocirugía y Psiquiatría, 36(4) ,190-194.

Ozonoff, S., Pennington, B. \& Rogers, S. (1996). Executive function deficits in high-functioning autistic children: Relationship to theory mind. Journal of Child Psychology and Psychiatry, 32, 1107-1122.

Pennington, B. \& Ozonoff, S. (1996). Executive functions and developmental psychopathology. Journal of Child Psychology and Psychiatry, $37,51-87$. 
Portellano, J. (2008). Neuropsicología infantil. Madrid: Síntesis.

Rapin, I. (1999). Autism in search of a home in the brain. Neurology, 52, 902-904.

Raven, J. (1984). Test de matrices progresivas para la medida de la capacidad intelectual. Manual para la aplicación. Buenos Aires: Paidós.

Rinehart, N., Bradshaw, J., Brereton, A. \& Tonge, B. (2002). A clinical and neurobehavioral review of high-functioning autism and Asperger's disorder. Australian and New Zealand Journal of Psychiatry, 36, 762-770

Romero-Munguía, M. (2002). Memoria procesal deficiente y alteraciones de la comunicación en la patogenia del autismo infantil. Revista Neurología, Neurocirugía y Psiquiatría, 35(4), 203-208.
Roselli, M., Matute, E. \& Ardila, A. (2010). Neuropsicología del desarrollo infantil. México D. F.: Manual Moderno.

Sattler, J. \& Hoge, R. (2008). Evaluación infantil: aplicaciones conductuales, sociales y clínicas. México D. F.: Manual Moderno.

Wechsler, D. (2005). Escala de inteligencia de Wechsler para niños-IV. Madrid: TEA.

Williams, D., Goldstein, G., Kojkowiski, N. \& Minshew, N. (2008). Do individuals with high functioning autism have the IQ profile associated with nonverbal learning disability? Research in Autism Spectrum Disorders, 2(2), 353-361.

Wing, L. (1981). Asperger's syndrome: A clinical account. Psychological Medicine, 11, 115-129.
Fecha de recepción: 4 de septiembre de 2013 fecha de aceptación: 27 de enero de 2015 


\section{Anexo 1 \\ Consentimiento informado}

\section{INTRODUCCIÓN}

A usted señor(a)

representante legal de lo estamos invitando a participar en un es-

tudio de investigación cooperativo entre el Grupo de Neurociencias y Comportamiento de la Universidad Pontificia Bolivariana (UPB) y algunas instituciones clínicas y educativas especializadas en el manejo de niños con síndrome de asperger y autismo de alto funcionamiento.

Primero, nosotros queremos que usted conozca que:

- La participación es absolutamente voluntaria.

- Esto quiere decir que si usted lo desea puede negarse a participar o retirarse del estudio en cualquier momento sin tener que dar explicaciones.

- Los beneficios que usted recibirá no son de tipo económico.

- Es importante que usted comunique al grupo investigador cualquier opinión o inquietud que presente sobre su participación en el estudio.

\section{INFORMACIÓN SOBRE EL ESTUDIO DE INVESTIGACIÓN}

A continuación se describirá en detalle en qué consiste el estudio de investigación. Antes de tomar cualquier decisión de participación, por favor, tómese todo el tiempo que necesite para preguntar, averiguar y discutir, con cualquiera de los investigadores del Grupo de Neurociencias y Comportamiento, los aspectos relacionados con este estudio.

\section{Propósito}

El propósito de este estudio es identificar las diferencias existentes entre la Escala de Inteligencia de Wechsler para Niños IV (WISC-IV) y el Test de Matrices Progresivas de Raven, en cuanto a la capacidad intelectual en una muestra de niños con síndrome de Asperger y autismo de alto funcionamiento. Para este estudio se evaluarán niños entre los 6 y 16 años de edad, pertenecientes a instituciones especializadas y que cuenten con un diagnóstico emitido por un profesional certificado. Para lograr este propósito se utilizará un protocolo de evaluación neuropsicológica en el cual se incluyen los siguientes instrumentos, Test de Matrices Progresivas de Raven (Escala Especial-para edades de 4 a 11 años) (Raven, 1983), que es un test destinado a medir la capacidad intelectual de forma no verbal, donde el sujeto describe piezas faltantes de una serie de láminas preimpresas. En esta prueba se pretende el uso de habilidades perceptuales, de observación y razonamiento analógico para deducir el faltante en la matriz y Escala de Inteligencia de Wechsler para Niños -IV (WISC-IV) (Weschler, 2005), que tiene como objetivo evaluar las capacidades cognitivas de niños y adolescentes con edades comprendidas entre los 6 y 16 años de edad. A partir de su aplicación se puede obtener una aproximación al funcionamiento cognitivo e intelectual del individuo. 


\section{Procedimiento}

Se ha llevado a cabo un proceso de selección en el cual los profesionales de la institución especializada en la que se encuentra su hijo/a lo han remitido para participar en el estudio y por ello se le está brindando la información relacionada con este; de igual forma, debe recordarse que cualquier inquietud que se presente puede ser solucionada por el grupo investigador. Se realizará una entrevista para obtener datos de identificación del niño y antecedentes de salud importantes. Luego, se procederá a la evaluación de su hijo; dicha evaluación se hará en dos sesiones: la primera con una duración aproximada de 120 minutos y la segunda de 60 minutos. La información que se obtenga de las sesiones servirá para comprender cuál es el desempeño cognitivo del niño en tareas como razonamiento abstracto, la habilidad de desarrollar y mantener estrategias de solución de problemas, memoria, efecto de la interferencia, atención sostenida, organización, memoria visual y habilidades perceptivas. Así mismo, esta información permitirá hacer una descripción detallada de la capacidad intelectual del evaluado. Si usted requiere un informe sobre los resultados de las evaluaciones del desempeño de su hijo y del proceso de intervención, puede solicitarlo por escrito y se le entregará. Este informe solo le será entregado a usted.

\section{Riesgos}

De acuerdo con la Resolución 008430 de 1993 del Ministerio de Salud, no existe ningún tipo de riesgo por hacer parte del estudio; esto quiere decir que durante su participación en el estudio su hijo/a no correrá ningún riesgo a nivel médico, ni se realizará ningún tipo de intervención clínica o procedimiento invasivo.

\section{Beneficios}

Los estudios de investigación como este producen conocimientos que pueden ser aplicados para la evaluación futura de otras personas, en otros contextos, con otras costumbres, condiciones sociales, características psicológicas, edades y niveles socioeconómicos. Por esta investigación ni usted, ni su hijo recibirán beneficio económico; sin embargo, si su hijo participa en el estudio será valorado e intervenido a nivel psicológico, lo cual le permite obtener una orientación con respecto a la conducta del menor y su evolución. Además, gracias a la contribución de personas como usted será posible que se puedan comprender mejor aspectos relacionados con los trastornos del espectro autista, específicamente con procesos de evaluación, manejo e intervención de los niños que lo padecen.

\section{Reserva de la información}

La información que usted aportará a través de los cuestionarios y la entrevista durante esta investigación será absolutamente confidencial y no será proporcionada a ninguna persona diferente a usted bajo ninguna circunstancia. A cada cuestionario se le asignará un código de tal forma que otras personas auxiliares en la investigación no tengan acceso a sus datos personales. Solo los investigadores (responsables del estudio) tendrán acceso al código y su identidad verdadera para poder localizarlo. En caso de que un auxiliar del grupo de investigación deba evaluar su hijo, este tendrá acceso a su identidad pero no conocerá el código de los resultados, para de esta manera garantizar su derecho a la intimidad. De igual forma, no se divulgarán, ni se publicarán a través de ningún medio los nombres de los participantes y 
nadie fuera del grupo investigador conocerá su información personal. Los síntomas y diagnósticos que se detecten solo le serán informados al padre si con ello puede conseguirse un tratamiento eficaz y a tiempo.

\section{Derecho a retirarse del estudio de investigación}

Es importante que usted tenga claro que puede retirarse del estudio en el momento que desee; sin embargo, los datos obtenidos hasta ese momento seguirán formando parte del estudio, a menos que usted solicite por escrito que su identificación e información sea borrada de nuestra base de datos. Al retirar su participación usted deberá informar al grupo investigador si desea que sus respuestas sean eliminadas; los cuestionarios que usted llenó pueden ser incinerados.

\section{Información médica no prevista}

Durante el desarrollo de este estudio es posible que se obtenga información acerca de la conducta de su hijo(a) no prevista. Si esta información se considera importante para el cuidado, el bienestar y la salud de su hijo, nosotros le recomendaremos el especialista adecuado y, en este caso, nuestra investigación NO cubre los costos de dicha atención. Si nosotros tenemos información relevante acerca de la conducta de su hijo, esta será suministrada de manera personal; así mismo, si usted obtiene información por parte de otros médicos de alguna enfermedad o aspectos importantes que no conocía antes de vincularse a este estudio, por favor, contáctenos, pues podría ser importante para nuestro estudio.

\section{ESTUDIOS FUTUROS}

Los resultados de esta investigación serán grabados con un código numérico y estos no serán colocados en la historia clínica del niño. Los resultados serán publicados en revistas de literatura médica científica, pero se garantiza que la identificación de los participantes no aparecerá en estas publicaciones. Es posible que en el futuro la historia clínica y los registros del desempeño de los niños sean utilizados para otras investigaciones cuyos objetivos o propósitos no aparecen especificados en este formato de consentimiento informado que usted firmará. Si esto llega a suceder, toda la información será entregada de manera codificada para garantizar que no se revelará el nombre del niño. De igual manera, si otros grupos de investigadores solicitan información para hacer estudios cooperativos, la información se enviará solo con el código, es decir, la identificación del niño no saldrá fuera de la base de datos del grupo de investigación.

\section{CONSENTIMIENTO INFORMADO}

Después de haber leído completamente toda la información contenida en este documento con respecto al proyecto: Autismo e inteligencia: comparación entre el desempeño en el WISC-IVy el desempeño en el Test de Matrices Progresivas de Raven en una muestra de niños con síndrome de Asperger y autismo de alto funcionamiento, habiendo dispuesto de tiempo suficiente para reflexionar sobre las implicaciones de mi decisión libre, consciente y voluntariamente manifiesto que he decidido autorizar la participación de de quien soy el representante legal

o tutor, dada su imposibilidad de firmar este documento con completa autonomía por tratarse de un menor de edad. 


\section{Paula fernanda Pérez Rivero, Lía Margarita Martínez Garrido}

Adicionalmente, autorizo que la información de la historia clínica y los registros del desempeño en tareas cognoscitivas, de mi representado legal, sean utilizados en otras investigaciones futuras.

En constancia, firmo este documento de consentimiento informado, en la ciudad de

$$
\text { el día __ del mes de __ del año }
$$

Nombre, firma y documento de identidad del padre, madre o representante legal:

Nombre: Firma:

Cédula de ciudadanía \#: de

Nombre, firma y documento de identidad del investigador:

Nombre: Firma:

Cédula de ciudadanía \#: de

Nombre, firma y documento de identidad del director del proyecto:

Nombre: Firma:

Cédula de ciudadanía\#: de 